\title{
A mesoscopic oxide fuel clustering and its global performance
}

\author{
Ruben Shaginyan, Valery Kolesov \\ Obninsk Institute for Nuclear Power Engineering of the National Research Nuclear \\ University MEPhI, Russia
}

Evgeny Ivanov

Institut de Radioprotection et de Sûreté Nucléaire (IRSN), BP 17, 92262 Fontenay-aux-Roses Cedex, France

shaginian@iate.obninsk.ru,kolesov@iate.obninsk.ru,evgeny.ivanov@irsn.fr

\begin{abstract}
Transient fuel behavior in a Light Water-cooled Reactor core depends on nuclear properties (Doppler broadening, moderation ratio, and, sometimes, neutron gas temperature etc.) and on variations of thermal-physics parameters (temperature distributions, fuel elongation and moderator density).

Usually, in a rough reactor analysis one ignores the very details of temperature distributions largely staying in a frame of so-called adiabatic assumptions (when temperature and density distribution are changing in sync keeping given spatial shapes). In majority of practical applications the radially distributed temperature fields are represented as monotonically smeared ones as if fissile and other materials are homogeneously mixed.

Moreover, no one measurement technique allows counting precise correlation between reactivity feedback and in-pellet temperature and materials space-time distributions.

However, if fuel is made of Mixed Oxide Plutonium-Uranium compound the behavior of Light Water Reactor would be impacted by an appearance of Pu-rich agglomerates that could be large enough to change physical processes.

In such case the fuel reacts on power and temperature variations no more as a homogeneous but a heterogeneous media (on a mesoscopic scale, of course).

It leads to changes in a fission product distributions, a fission gas release and, even, to an appearance of multiple components in a Fuel Temperature Coefficient and in a Power Reactivity feedback.

These components would depend non-linearly on power, power rate and on some details of a heat transfer.

This paper is the only first step of a broad research program where we are estimating the relevant phenomena just by an order of magnitude.
\end{abstract}

KEYWORDS: MOX, Doppler, transient analysis

\section{INTRODUCTION}

Considering one or another phenomena of interest one used to apply certain kinds of assumptions and simplifications keeping, mainly, the important (from some points of view) parameters of a nuclear power system under consideration. It should be ensured that such assumptions and caused by them simplifications are reasonable with respect to given Target Accuracy Requirements (TARs). 
These TARs and ignorance criteria are inferred on a past experience basis. However conjuncture is changing and what we ignored in the past would play a notable role now and in a nearest future.

For example, in mixed or deeply burned fuel there are, among other, some peculiarities caused by a separation of fissile and other materials on a mesoscopic scales. Physically talking a heat generating fraction doesn't coincide with a heat conducting ones that lead to changes in, both steady-state and transient, fuel behavior. Such phenomenon has been observed while use a vibropac Mixed Oxide (MOX) fuel where Pu-rich zones agglomerate reached size of $\sim 10 \div 100 \mathrm{~m}[1,2,3]$. The impact on steady-state characteristics has been identified and quantified in different publications $[1,2,4]$. We would complement these studies focusing on transient aspects. In such a context one may identify the following questions to be addressed.

1) how far such heterogeneity impacts the power feedbacks and other parameters of given system;

2) if yes, would it be needed to apply another approach to characterize a power feedback; and

3) how far such phenomenon could have an effect on various Plants Measurements and Observations and on experiments in Fuel Test Reactors.

There is no doubts answering affirmatively on the first question since the internal heterogeneity has an influence on, both local and global, temperature distributions.

Moreover, it is easy to demonstrate an, even, inversion of temperature field stretched on Pu-rich agglomerates which impacts an "effective fuel temperature".

The "effective temperature" means a synthetic variable to be used in a lumped parameter model representing nuclear fuel status in a transient. The lumped model, as such, operates with such values as Fuel Temperature Coefficient (FTC) and power reactivity feedback (PRF). FTC, in its order, is parametrized by the "effective temperature". So, in case of variations or, even, inversion of one or another component of temperature distribution one should change the "effective temperature" revising the very basis of FTC parametrization.

In the article we are discussing some peculiarities of transients caused by an existence of Pu-rich agglomerates in fuel matrix [1,2]. As a result we had to split the Doppler part of FTC on two components (associating it with Pu-reach particles and with other oxide matrix) in order to keep (roughly, of course) the physics of transient and of power response in MOX loaded core. Of course, in this paper we have no opportunity to present all what might be needed to characterize the transient behavior since it exceeds the scope of reactor physics domain requiring comprehensive multi-physics considerations.

Of course, this work should be considered as a small part of a broad research program on a study of core and fuel performance. Nevertheless, it seems sufficient to give some semi-qualitative assessments as follows:

1) an assessment whether or not the temperature fields might be distributed in opposite direction: normal one descending from the center to periphery will be for the uranium oxide matrix and inversed one will be for the ensemble of Pu-rich particles; and

2) a demonstration of an influence on a core behavior applying such FTCs to an internationally available exercise on an LWR transient.

Paper comprises a general reasoning concerning some peculiarities of MOX loaded systems modeling in terms of transient phenomena of interest, demonstrating an influence of such two-component approximation on power spike and deposit energy in a Reactivity Insertion transient exercise derived from one OECD-NEA UAM-LWR benchmark ${ }^{1}$.

More general one may talk about a preliminary analyzing (by the order of magnitude) emerging multiphysical phenomena caused by fuel materials relocation on a mesoscopic scale.

\section{GENERAL REMARKS AND MAJOR STATEMENTS}

As said, an internal heterogeneity could contribute in the entire core behavior. It might impact almost all components of power feedbacks while the most impacting one would be FTC and, namely, Doppler-

\footnotetext{
${ }^{1}$ OECD-NEA means the Nuclear Energy Agency (NEA) is a specialized agency within the Organization for Economic Co-operation and Development (OECD) while UAM-LWR (an Uncertainty Analysis in a Modelling of Light Water cooled Reactors ) is one of the NEA projects.
} 
effect variations. There are also notable changes in fission products and fuel materials distributions and, as a consequence, in a fission gas release, not only the changes of reactivity feedbacks. However, since the conference is dedicated to a reactor physics we are focusing on the Doppler-effect as a physical phenomenon considering it as a product of the domains of particle transport, criticality, thermohydraulics and mechanics.

Note that the Doppler-effect in comparison with others gives an instant response on a power variations being well-understood and elaborated with respect to its principal components despite on certain uncertainties reminded at the level of nuclear data and thermodynamics modeling [5].

As said, Doppler-effect is parametrized for practical applications using one single "effective" temperature and one kind of dependence of reactivity on such temperature. There are many publications on elaboration and optimization of these approximated "effective" temperatures [6].

However, in a case of mentioned above internal heterogeneity, like for MOX fuel, an approximation, as a process, could face additional complexities in dependence on size and other properties of $\mathrm{Pu}$-rich or other agglomerates that might be a size between $\sim 5,10$ or even $25 \mathrm{~m}$.

Here, talking about the only Doppler-effect we are basing our studies on a set of numerical simulations considering them as numerical experiments. The studies include the following steps.

1. The first step is a quantification of an approximated coefficients to characterize Doppler-broadening effect implying that there would be relevant to one or another reduced order surrogate model of transient; the surrogate model seems to be unavoidable because estimating reactivity coefficients we are dealing with small perturbations, i.e. the values of the second order of magnitude.

2. The second step includes an exact calculation of distributions of temperature and power profiles in a heterogeneous system. Such study will be done iteratively in order to take into account local temperature distributions and local reactivity feedbacks whether in a traditional or a corrected representation of a structure of a fuel pellet.

3. At the third step we are combining exact calculations and surrogate modeling we are performing comparative studies and demonstrations of the principle mechanisms that drive a power reactivity feedback in a mixed fuel.

It is not necessary to consider the whole reactor core for the analysis of the Doppler-effect. We are staying over there on a Doppler-effect within a pin cell modeling, which is the smallest possible unit cell in a nuclear reactor. Although the core behavior in transients is driven by all factors we can limits our consideration the only on Doppler-effect since others have no direct dependence on mesoscopic fuel pellet structures. Such an analysis gives an idea of the importance of different contributions and could be sufficient illustrating the major statements.

\section{MODELS AND SIMULATIONS}

Coming to modeling and parametrization we should remain that, as said, there are many reasons why feedbacks are considered as elements of a lumped dynamic model. The term lumped means weighted aggregation of field functions where weights depend on physics behind and a further application of one or another parameter. Thus, for the temperature feedbacks we sued to use some "effective" temperatures which the most popular one is given by the following G. Rowlands [6] formulae:

$$
T_{\text {eff }}=4 \cdot T_{C T L} / 9+5 \cdot T_{\text {edge }} / 9
$$

where $\mathrm{T}_{\text {eff }}, \mathrm{T}_{\mathrm{CTL}}$ and $\mathrm{T}_{\text {edge }}$ are effective temperature, fuel central line temperature and a temperature at a periphery of fuel pellet, respectively. Of course, it is the only approximation but it seems sufficient for the main practical needs. However, there might be some exceptions like we would consider below. The approximation given by Equation 1 presumes the continuous temperature distribution with maximum in the center and minimum at the edge of the pellet (see Figure 1a) while, for example, in a case of an appearance of Pu-rich agglomerated the temperature would be even inversed in comparison with the traditional imagination (see Figure 1b). 
Looking at Figure 1 one can distinguish two temperature fields: traditional continuous one distributed in an uranium oxide matrix and point wise "distribution" smeared on a surface "stretched" on Pu-rich particles.

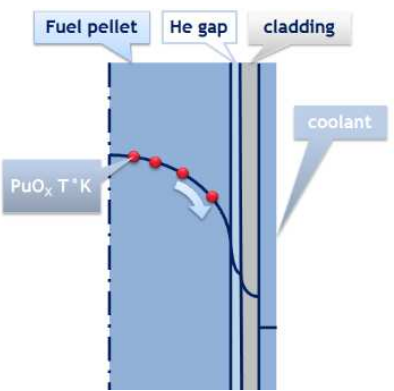

a $\mathrm{b}$

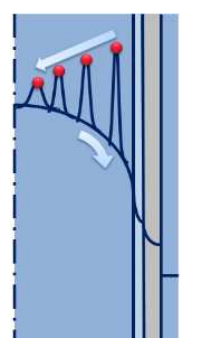

Figure 1: Temperatures in a homogeneous (a) media and in one with fissile agglomerates (b).

Note, please, that such "inversion" looks paradoxical only if fuel looks like a two-dimensional object where thermal flux flows along the only one direction from the center to the edge. Our reality, of course, is three-dimensional one and the heat generated in the particles (agglomerates) follows the flux but not pass of cooling. This is why particles will have higher temperature than uranium oxide matrix and the field "stretched" on their ensemble will demonstrate an inversed temperature radial dependence. Such difference will be more pronounced in a transient, of course.

As said, our objective was not to provide all quantified assessments but the only to consider the relevant phenomena by an order of magnitude.

We've decoupled the model dividing thermal-flow and particle transport numerical analysis on the basis of a pin-cell model using ANSYS Fluent [7], and SERPENT-2 [8] tools.

The geometry for calculations was simplified reducing the object to a multi-annular radial division (see Figure 2). Each zones has been filled by Pu-rich spherical particles that are describe below.

In our calculational scheme the Pu-rich particles were randomly distributed within each concentric geometrical zone (see Figure 2a). These particles of Pu-rich materials were postulated to be spherical ones with a uniformed dimension.

Such geometry allowed performing simulations of power distribution and, that is more important, of importance function (Figure $2 b$ ) facilitating parametrization of the FTC.

Some available Monte-Carlo codes, including SERPENT-2, have such essential for our studies options like adjoint-weighted perturbation theory to compute in a stringent manner small reactivities and geometrical modules adopted to simulate randomly placed fuel particles. Both SERPENT options were used in the numerical studies.

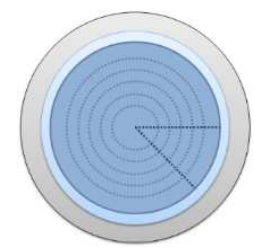

a

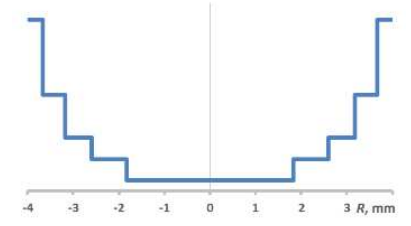

b

Figure 2: Geometrical model (a) and aggregated material worth radial distribution (b)

Review [1] proposes the following model assessing an effect of agglomerated plutonium oxide in the oxide matrix (see Figure 3a). The typical size of agglomeration are around $25 \mu \mathrm{m}$ which seems sufficient to reveal an effect of a resonance self-shielding.

Although, according to an available bibliography the plutonium oxide agglomerates would be of $\sim 10$ to $25 \mathrm{~m}$ diameter one size we smeared them within uranium oxide placed nearby (see Figure $3 \mathrm{~b}$ ). Postulating the average size of Pu-rich particles of $\sim 10 \mathrm{~m}$ we smeared each one in a homogeneously mixed zone of oxides $\left(\mathrm{PuO}_{1.6}\right.$ and $\left.\mathrm{UO}_{2.1}\right)$ sized on around $0.1 \mathrm{~mm}$. 


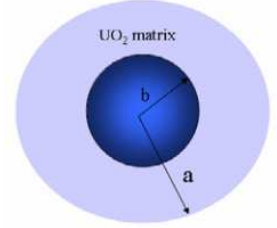

a

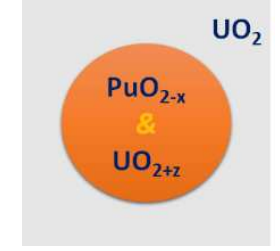

$\mathrm{b}$

Figure 3: Spherical cell model for the $\mathrm{PuO}_{2} / \mathrm{UO}_{2}$ system as in [2] (a) and in a current work (b).

Computed parametrical dependences have been represented by an exponentiation with a temperature as a basis and an exponent dependent on which kind of resonances form reactivity feedback. In our case major influential cross section in matrix and in Pu-rich zones are different.

We used traditional definition and approximation of Doppler reactivity feedback defining the reactivity as follows:

$$
\rho(T)=\frac{k_{e f f}\left(T_{0}\right)-k_{e f f}(T)}{k_{e f f}\left(T_{0}\right) \cdot k_{e f f}(T)},
$$

where $\rho(T), T, T_{0}, k_{e f f}(T)$ and $k_{e f f}\left(T_{0}\right)$ are temperature dependent reactivity, actual and initial temperature, and multiplication factors at actual and initial temperatures, respectively, and the approximation as a kind of exponential function:

$$
\rho(T) \approx\left[C_{1} \cdot T_{0} / 1-\gamma\right] \cdot\left[\left(T / T_{0}\right)^{\gamma}-1\right]+C_{2},
$$

where all notations are the same as above and $\gamma, C_{1}$ and $C_{2}$ are parameters to be adjusted in a physical or a numerical experiments. That leads to the simplified formulae like the following:

$$
\frac{d \rho}{d T} \approx \frac{K_{D}}{T^{\alpha}}
$$

where $\rho, T, K_{D}$ and $\alpha$ are reactivity, temperature (in absolute units), Doppler coefficient and power coefficient.

Despite the differences in temperature distributions for an ensemble of Pu-reach particles and other matrix the inferred Doppler feedback coefficients were finally parametrized as a function of uranium oxide effective temperature given by G. Rowlands [6]. The fitting procedure gave us the following dependences: for the uranium oxide matrix

$$
\frac{d \rho}{d T} \approx \frac{K_{U O x}}{T^{0.5}}
$$

where $\rho, T, K_{U O X}$ and $\alpha=-0.5$ are reactivity, temperature (in absolute units), Doppler coefficient and for Pu-reach component

$$
\frac{d \rho}{d T} \approx \frac{K_{P u O x}}{T^{0.6(6)}},
$$

where $K_{P u O x}$ and $\alpha=-0.6(6)$ are a Doppler coefficient and power of an exponent fitted to Pu-rich zones. Estimated quantitative coefficients are given in Table I. 
Table I: Doppler-based components of FTC due to uranium oxide and Pu-rich zones

\begin{tabular}{|l|l|l|}
\hline & $\mathrm{K}_{\mathrm{D}}, \mathrm{pcm}$ & comment \\
\hline due to $\mathrm{UO}_{2}$ matrix & -10.6 & power constant $\sim-0.5$ \\
\hline due to $\mathrm{Pu}$-rich zone & 0.9 & power constant $\sim-0.667$ \\
\hline
\end{tabular}

Note, that in a steady state these coefficients give a linearized FTC of $\sim-2.987 \mathrm{pcm} /{ }^{\circ} \mathrm{C}$ that corresponds to traditionally applied coefficients [1].

Such representation is very useful in terms of reactor physics measurements but not necessarily in transient simulations. For the latest one could be more convenient to parametrize them in terms of power feedback. Although the numerical studies encompassed a broad set of scenarios hereinafter we would illustrate the phenomena are presenting the only case taken from UAM-LWR [9] benchmark where UOX fuel elements.

Honestly, we a-priory expected an increase of power spikes in transient as it could be illustrated by comparison of the simplified dynamic models (Equations 6 and 7). Traditional point-wise dynamic model looks like the following:

$$
\left\{\begin{array}{c}
\frac{d q}{d u} \approx \frac{\rho_{E X T}+\rho_{P W R}(q)+\cdots-}{\Lambda_{E F F}} \cdot q+\sum_{i} \lambda_{i} \cdot C_{i} \\
\frac{d C_{i}}{d u} \approx \frac{\beta_{i}}{\Lambda_{E F F}} \cdot q+\lambda_{i} \cdot C_{i} \\
\frac{d T}{d u} \approx \frac{q}{\tau_{p i n}}-\theta \cdot\left(T-T_{0}\right)-\cdots \\
\cdots
\end{array},\right.
$$

while two-component power reactivity feedback transforms the traditional form onto higher order equations like the following one

$$
\frac{d q}{d u} \approx \frac{\rho_{E X T} \cdot q}{\Lambda_{E F F}-\gamma_{R A T E} \cdot q}+\frac{\gamma_{P W R} \cdot q^{2}}{\Lambda_{E F F}-\gamma_{R A T E} \cdot q}+\cdots
$$

where $q, \Lambda_{E F F}, \beta_{e f f}, \rho_{E X T}, \gamma_{P W R}$ and $\gamma_{R A T E}, C_{i}$ and $\lambda_{i}$ are fission power, neutron generation lifetime, delay neutron effective fraction, externally inserted reactivity, power reactivity coefficient and transient power feedback coefficient, delay neutron concentrations and delay neutron decay constant.

The inserted reactivity has been reduced in comparison with the benchmark specification as far as MOX fueled rector has lower delay neutron effective fraction.

It is easy to see that such approximation is one of the most unstable ones and looks like the model where neutron effective generation lifetime depends on power. The results depicted on Figure 4 characterize as power evolution (a) and correspondent deposited energy (b) during the transient

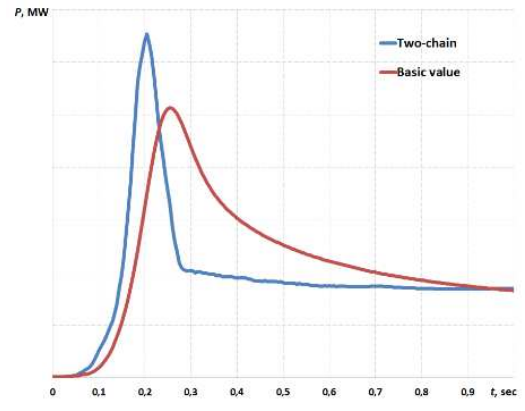

a

Figure 4: Transient Power (a) and Deposit Energy (b) evolution in the models with a traditional homogenous (red) and a heterogeneous placement of fissile clusters (blue) 
In addition, one should note that in this transient an evolution of temperature and neutron distributions dominated over all other phenomena like ones relayed to fuel pins' elongation, assembly deformations and so on.

\section{DISCUSSION}

Modern tendency in nuclear technology development can be traced in a growing of a role of predictive comprehensive simulations in nuclear concepts design, engineering and safety assessment and, even in an operational support.

First of all these simulations are aimed to fill the gaps in relevant experimentations and in a feedbacks from operational experience while both (experimental capacities and exploitation background) are sufficiently limited.

The second stimulating reason is in a progressive development of algorithms and tools of multi-physics and multi-scale modeling along with an increasing of computational capacities.

Of course, there is no idea to replace objective observations by numerical experiments but to complement them. We should talk in such a context about a paradigm shift in a technology science where instead of an exact testing, especially in the domains where it seems fundamentally impossible, one could rely on a kind of implicit approaches where the data of interest could be somehow inferred from indirect measurements and extrapolated onto a domain of interest.

Such approaches necessarily require deep understanding of all complex phenomena of interest, where the term "understanding" means, amid others, an availability of adequate mechanistic model of the phenomena of interest.

Although the impacts due to certain peculiarities of nuclear fuel on a mesoscopic scales could appear in numerous applications this paper demonstrates the only limited ones. As said, being a part of more broad research program the work presented over here doesn't encompass all challenging impact factors to be taken into account in MOX loaded core and fuel behavior. Furthermore, we are discussing here the only impact due to internal heterogeneity of $\mathrm{Pu}$-contain fuel elements and focusing only on reactivity driven transients ignoring other fully multi-physics items. Others would be elaborated and presented later.

It should be also noted that in addition to Pu-reach agglomerations influence on a reactivity-driven core behavior they could impact fuel performance in a loss of cooling accident. Indeed local temperature of $\mathrm{Pu}$-rich zones becomes higher than other matrix while empiric coefficients of fission gas and helium retention in a fuel matrix are parametrized against the smeared temperature. Such local temperature, though, might not be measured in an exact experiments and observations but should be extrapolated using mechanistic modeling.

And, last but not least, one of the most challenging impacts of an internal heterogeneity could appear in a material testing experiments. This effect, of course, could definitely impact Fuel Tests experiments performed in a Transient Reactor Facilities (TRF) [10] complication an extrapolation of a Coupling Power Factor and, especially, a Transient Correction Factors [5].

Thus the domains where a consideration of the phenomena driven at the mesoscopic scale would be much broader than it has been demonstrated in the paper.

\section{CONCLUSIONS}

The domains of fuel and nuclear reactor behaviors are tightly coupled ones requiring for their simulations fully merged physical models and algorithms to bring an adequacy to reflections and representation of the relevant phenomena and their mechanisms. Such modeling necessarily includes both multi-physics and multi-scale phases while the current paper presents the only small portion of semi-decoupled analysis of an impact on core behavior due to some details of a fuel pellet structure.

Each of basic phenomena underpinning the system response are formed by several factors of different nature. For example, such well-known component of Fuel Temperature feedback as Doppler-effect is composed by resonance cross sections broadening or shrinking with temperature growing or reducing correspondently, while the temperature and its special distribution follow a misbalance between heating and cooling caused, in its order, by the power change. 
Thus, the effect, as such, includes several physics and spatially spanned fields like temperature and so on. In other words, the phenomena underlie it are fundamentally of multi-physics ones.

One of the domains - fuel performance one - as it is well-known has very complex relationships between different physics, evolution of the fuel pellet microstructure and change in the material properties, and reactor conditions. To overcome these fundamental complexities, simulations involves a lot of empirical or semi-empirical models, with parameters fit to experimental in-pile and out-of-pile fuel samples tests.

Actually, we focused on a consideration of how impacting might be an existence Pu-rich agglomerations in MOX fuel with respect to a transient behavior of LWR cores.

To do it we used available published data on MOX technologies, reactor analysis and one transient standard problem derived from UAM-LWR benchmark. Calculations have been performed using the following single- and low-physics tools: (1) SERPENT-2 for pure criticality and particle transport simulations, and (2) ANSYS for mechanics and thermo-flow analysis.

One could summarize the major findings as follows:

1) as it has been expected, computations by ANSYS demonstrated that the values of temperatures along an ensemble of Pu-rich agglomerates follow the heat generation distribution growing from the center to periphery of fuel pellet, i.e. in an opposite direction than for other fuel matrix;

2) consequently, FTC splits on two terms: the first one - positive - associated with Pu-rich agglomerates and the second one - negative - that represent other fuel matrix;

3) a two-component FTC leads to an increasing of power-peak in one postulated reactivity-driven transient and a reducing of deposit energy during the transient.

One should remain that the work was not aimed to address all relevant issues but to the only demonstrate their existence. We would like to stress that nowadays reactor physics items exceed traditional singlephysics domain becoming in an increasing extent a part of multi-physics one. So the results presented here should be considered as a preliminary ones while more relevant and consistent would be available at the end of more vast multi-physics research programs realizations.

\section{REFERENCES}

1. Ali R. Massih, Models for MOX fuel behavior A selective review SKI Report 2006:10, ISSN 11041374, ISRN SKI-R-06/10-SE, January 2006

2. Masayoshi Ishida, Yoshio Korei, Modeling and parametric studies of the effect of Pu-mixing heterogeneity on fission gas release from mixed oxide fuels of LWRs and FBRs, Journal of Nuclear Materials, Volume 210, Issues 1-2, 1994, Pages 203-215

3. Ian Crossland, "Nuclear Fuel Cycle Science and Engineering", A volume in Woodhead Publishing Series in Energy, Book • 2012

4. Luka Snoj, Ivan Kodeli \& Igor Remec (2014) Evaluation of the KRITZ-2 Criticality and Reaction Rate Benchmark Experiments, Nuclear Science and Engineering, 178:4, 496-508

5. Edited by Dan Gabriel Cacuci, Handbook of Nuclear Engineering, Springer, Boston, MA, 2010

6. G. Rowlands, Resonance absorption and non-uniform temperature distributions (1962) Journal of Nuclear Energy. Parts A/B. Reactor Science and Technology, 16 (4), pp. 235-236

7. ANSYS, Inc. (2016) ANSYS Fluent User's Guide, Release 17.2

8. Leppänen, J., et al. (2015) "The Serpent Monte Carlo code: Status, development and applications in 2013." Ann. Nucl. Energy, 82 (2015) 142-150

9. J. Hou et al., 'Benchmark for Uncertainty Analysis in Modelling (UAM) for Design, Operation and Safety Analysis of LWR, Phase III', NEA/NSC/DOC(2019), April 2019

10. D. Devin Imholte, Fatih Aydogan, Comparison of nuclear pulse reactor facilities with reactivityinitiated-accident testing capability, Progress in Nuclear Energy, Volume 91, 2016, Pages 310-324 